\title{
ELECCIONES MUNICIPALES EN LA RAAN
}

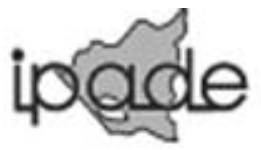

Instituto para el Desarrollo de la Democracia

La observación electoral es un elemento sustantivo para fortalecer la credibilidad de los electores en el sistema electoral nicaragüense partidarizado, y preservar la validez de los mecanismos cívicos en la alternabilidad del poder es esencial para la viabilidad del sistema democrático. Luego de las elecciones municipales realizadas en 146 municipios del Pacífico y Centro de Nicaragua el 9 noviembre del 2008, un porcentaje importante de los electores y dirigentes liberales han acusado al Consejo Supremo Electora (CSE), al partido de gobierno y a la dirigencia del Partido Liberal Constitucionalista (PLC) de coludirse para hacer un fraude en los municipios donde el Movimiento Vamos Con Eduardo tenía posibilidades de ganar estas plazas. Los cuestionamientos a los resultados electorales en al menos treinta municipios fueron avalados incluso por magistrados del propio CSE, provocando una crisis en la cual diversos sectores del país, Iglesia, Sector Privado, organismos de Sociedad Civil, PLC y Cooperación Internacional, demandaran un recuento que a la fecha no ha tenido respuesta institucional.

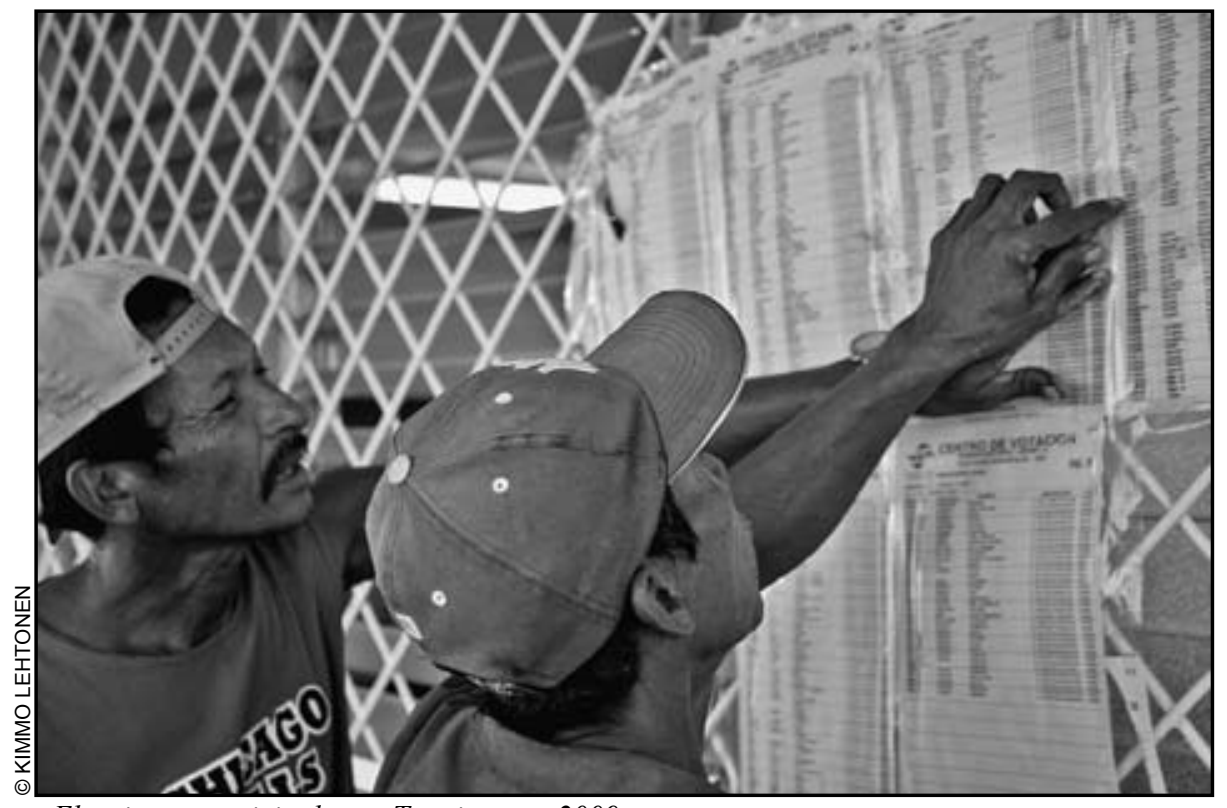

Elecciones municipales en Tuapi, enero 2009.
Para el 18 de enero del 2009 se programó las elecciones municipales en siete municipios de la RAAN, las cuales se realizaron con el temor de sectores costeños que las irregularidades y falta de transparencia del Pacífico se pudiesen repetir.

IPADE ha valorado la importancia de nuestro derecho constitucional de participación ciudadana observando las elecciones, con o sin acreditación del CSE; por ello, y a solicitud de los partidos políticos de la Costa Caribe, de lideres comunitarios y religiosos y de los Alcaldes, de observar estos comicios, dispuso una red de 300 observadores(as) en seis de los siete municipios de la RAAN, para verificar la transparencia o no de las elecciones.

IPADE dispuso de estrategias y metodologías de observación utilizadas en países no democráticos o de reciente transición hacia la democracia, y se implementan observaciones móviles en cada centro de votación que permiten, aun cuando no se está dentro del recinto de votación, tener una percepción del desarrollo de las elecciones y valorar si el proceso tiene las garantías mínimas de transparencia para ejercer el sufragio por los electores y si se respeta la voluntad popular expresada en las urnas.

Para ello organizamos seis Centros Municipales de monitoreo, en los municipios de: Siuna, Rosita, Bonanza, Mulukuku, Waspán y Puerto Cabezas, y un centro nacional de procesamiento en Managua. Nuestra red tuvo presencia en 121 Centros de Votación, el 48\% de los habilitados por el CSE, con una cobertura del 100\% en los Centros de Votación Urbanos y en el área rural del $35 \%$. 
Tala. 1 Cobertura de Centros de Votación por IPADE

\begin{tabular}{|c|c|c|c|c|c|c|c|c|c|c|}
\hline \multirow[b]{2}{*}{ No } & \multirow[b]{2}{*}{ Municipios } & \multicolumn{3}{|c|}{ Total CV del CSE } & \multicolumn{6}{|c|}{ Cobertura del Monitoreo de IPADE } \\
\hline & & URB & RUR & TOTAL & URB & $\%$ & RUR & $\%$ & TOTAL & $\%$ \\
\hline 1 & WASPAM & 3 & 49 & 52 & 3 & 100 & 10 & 20 & 13 & 25 \\
\hline 2 & Puerto Cabezas & 17 & 35 & 52 & 17 & 100 & 14 & 40 & 31 & 60 \\
\hline 3 & Rosita & 9 & 17 & 26 & 9 & 100 & 7 & 41 & 16 & 62 \\
\hline 4 & BONANZA & 5 & 14 & 19 & 5 & 100 & 8 & 57 & 13 & 68 \\
\hline 5 & Siuna & 13 & 56 & 69 & 13 & 100 & 28 & 50 & 41 & 59 \\
\hline 6 & MULUKUKú & 2 & 17 & 19 & 2 & 100 & 5 & 29 & 7 & 37 \\
\hline 7 & PRINZAPOLKA & 0 & 16 & 16 & & & & & & 0 \\
\hline & TOTAL & 49 & 204 & 253 & 49 & 100 & 72 & 35 & 121 & 48 \\
\hline
\end{tabular}

* Elaboración propia de IPADE, utilizando Plataforma Electoral del CSE.

\section{Constitución y Apertura de 260 JRV (51\%).}

Según los reportes de las zonas urbanas y rurales, los materiales electorales llegaron al $99 \%$ de las JRV, solo se reportan tres casos donde llegaron tarde, y el proceso de votación inició con demoras.

El proceso de votación en la RAAN inició sin mayores incidencias, con la apertura del 100 por ciento de las 260 Juntas Receptoras de Votos reportadas por nuestra red; en la constitución de las mismas se garantizó la presencia de fiscales, y la votación se inició con una buena afluencia de electores en los Centros de Votación en la hora establecida.

$>$ De acuerdo a nuestros informes municipales, en esta parte del proceso no se reportaron problemas para ejercer la labor de observación.

En un $99 \%$ de las JRV los fiscales presenciaron el proceso de Constitución y Apertura, se reportó tres casos de JRV en el municipio de Puerto Cabezas, donde a los fiscales se les expulso de la JRV, por llegar tarde.

\section{Desarrollo del Proceso de Votación.}

Durante el desarrollo de la Votación, nuestra cobertura se incrementó hasta 298 JRV, en las que se obtuvo información de nuestros observadores sobre el desarrollo de esta parte del proceso.
En 26 JRV (9\%) se dieron situaciones que interrumpieron el proceso de votación momentáneamente, estos son los casos:

- En 10 JRV reuniones de grupos, en 7 JRV disturbios, en 5 JRV hubo propaganda política, en 3 JRV se les negó ingreso a fiscales, y en una sola JRV, durante el día se expulso a un fiscal de un partido político.

\section{Cierre del Proceso de Votación.}

- Un $23 \%$ de las JRV cerraron antes de las 6 pm.; el $64 \%$ de las JRV cerraron entre las 6 y las 8 de la noche; sólo un $13 \%$ cerró después de las 8 de la noche.

- En un $96 \%$ de las JRV se atendieron a los ciudadanos que asistieron a ejercer su derecho al voto, únicamente en un $4 \%$ quedaron ciudadanos $\sin$ votar.

- En un $53 \%$ de las JRV hubo presencia de Policías Electorales dentro de los recintos de votación, en violación a la ley. El restante $47 \%$ de JRV, los policías electorales estuvieron fuera de los locales, en cumplimiento de la ley electoral.

\section{Escrutinio.}

- En un $77 \%$ de JRV se reporta presencia de al menos tres o más fiscales, en un $13 \%$ la presencia de dos fiscales, y solamente un $4 \%$ la de un solo fiscal. 
- En el $96 \%$ de las JRV se cumplió con la obligación de dar a conocer los resultados del escrutinio, pegando los resultados en la parte externa de las JRV.

\section{Resultados Parciales por Municipio de la observación de IPADE}

Los datos del presente informe se refieren a Resultados para Alcaldes y Vice Alcaldes tomados directamente por nuestra red de observadores y procesados por nuestros centros de cómputos municipales y luego a nivel nacional. A continuación se presentan resultados obtenidos por cada partido y/o alianzas de partidos y la cantidad de JRV reportadas en cada municipio.

\section{PUERTO CABEZAS: 86 JRV Reportadas.}

Tabla.2

\begin{tabular}{|l|c|c|}
\hline $\begin{array}{l}\text { Partidos y/o } \\
\text { Alianzas de } \\
\text { Partidos }\end{array}$ & $\begin{array}{c}\text { Votos válidos } \\
\text { por partido }\end{array}$ & $\%$ \\
\hline PLC & 3,867 & $\mathbf{3 0}$ \\
\hline FSLN & 4,678 & $\mathbf{3 7}$ \\
\hline PAMUC & 120 & $\mathbf{1}$ \\
\hline PRN & 50 & $\mathbf{0 . 4}$ \\
\hline YATAMA & 3,543 & $\mathbf{2 8}$ \\
\hline ALN & 506 & $\mathbf{4}$ \\
\hline AC & $\mathbf{1 4}$ & $\mathbf{0 . 1}$ \\
\hline
\end{tabular}

* Elaboración propia de IPADE

Estos datos recopilados por la red de observadores de IPADE coinciden con la información del Centro de Cómputo Municipal de Bilwi (Puerto Cabezas), trasmitidas al Centro nacional de Cómputo, la mañana del 19 de enero, que daba cuenta de las juntas urbanas y algunas comunidades. Nuestra red reporta 12,778 votos válidos, y el Centro de Cómputo habla de 13,203 , lo que refleja una diferencia mínima.

Del cuadro anterior se desprende que los resultados de la votación que nuestros observadores obtuvieron coinciden con la tendencia que ha publicado el CSE lo que indica que la voluntad popular expresada en las urnas ha sido respetada.

Tabla. 4
Tabla. 3 Comparativo del CSE vrs. Observación de IPADE en el Municipio de Puerto Cabezas

\begin{tabular}{|c|c|c|c|c|}
\hline \multirow{2}{*}{$\begin{array}{l}\text { Partidos } \\
\text { Políticos }\end{array}$} & \multicolumn{2}{|c|}{$\begin{array}{l}\text { Datos recopilados } \\
\text { por la Red de IPADE } \\
\text { con } 86 \text { JRV }\end{array}$} & \multicolumn{2}{|c|}{$\begin{array}{l}\text { Datos del CCM en } \\
\text { Bilwi con } 82 \text { JRV }\end{array}$} \\
\hline & $\begin{array}{l}\text { Votos } \\
\text { Válidos }\end{array}$ & $\%$ & $\begin{array}{l}\text { Votos } \\
\text { Válidos }\end{array}$ & $\%$ \\
\hline PLC & 3867 & $30 \%$ & 3909 & $30 \%$ \\
\hline FSLN & 4678 & $37 \%$ & 4842 & $37 \%$ \\
\hline PAMUC & 120 & $1 \%$ & 101 & $1 \%$ \\
\hline PRN & 50 & $0.4 \%$ & 57 & $0.4 \%$ \\
\hline YATAMA & 3543 & $28 \%$ & 3776 & $29 \%$ \\
\hline ALN & 506 & $4 \%$ & 502 & $4 \%$ \\
\hline$A C$ & 14 & $0.1 \%$ & 16 & $0.1 \%$ \\
\hline Totales & 12,778 & & 13,203 & \\
\hline
\end{tabular}

* Elaboración propia, en base a datos comparados de la observación en CV y el Centro de Cómputo del CSE en Puerto Cabezas.

MULUKUKÚ: 27 JRV Reportadas

Hay una ventaja para el PLC sobre el FSLN.

\begin{tabular}{|l|c|c|}
\hline $\begin{array}{c}\text { Partidos y/o Alianzas } \\
\text { de Partidos }\end{array}$ & $\begin{array}{c}\text { Votos válidos por } \\
\text { partido }\end{array}$ & $\%$ \\
\hline PLC & 897 & $\mathbf{4 1}$ \\
\hline FSLN & 878 & $\mathbf{4 0}$ \\
\hline PAMUC & 3 & $\mathbf{0 . 1}$ \\
\hline PRN & 52 & $\mathbf{2}$ \\
\hline YATAMA & - & $\mathbf{0}$ \\
\hline ALN & 347 & $\mathbf{1 6}$ \\
\hline AC & $\mathbf{1}$ & $\mathbf{0 . 0 5}$ \\
\hline
\end{tabular}

* Elaboración propia de IPADE 


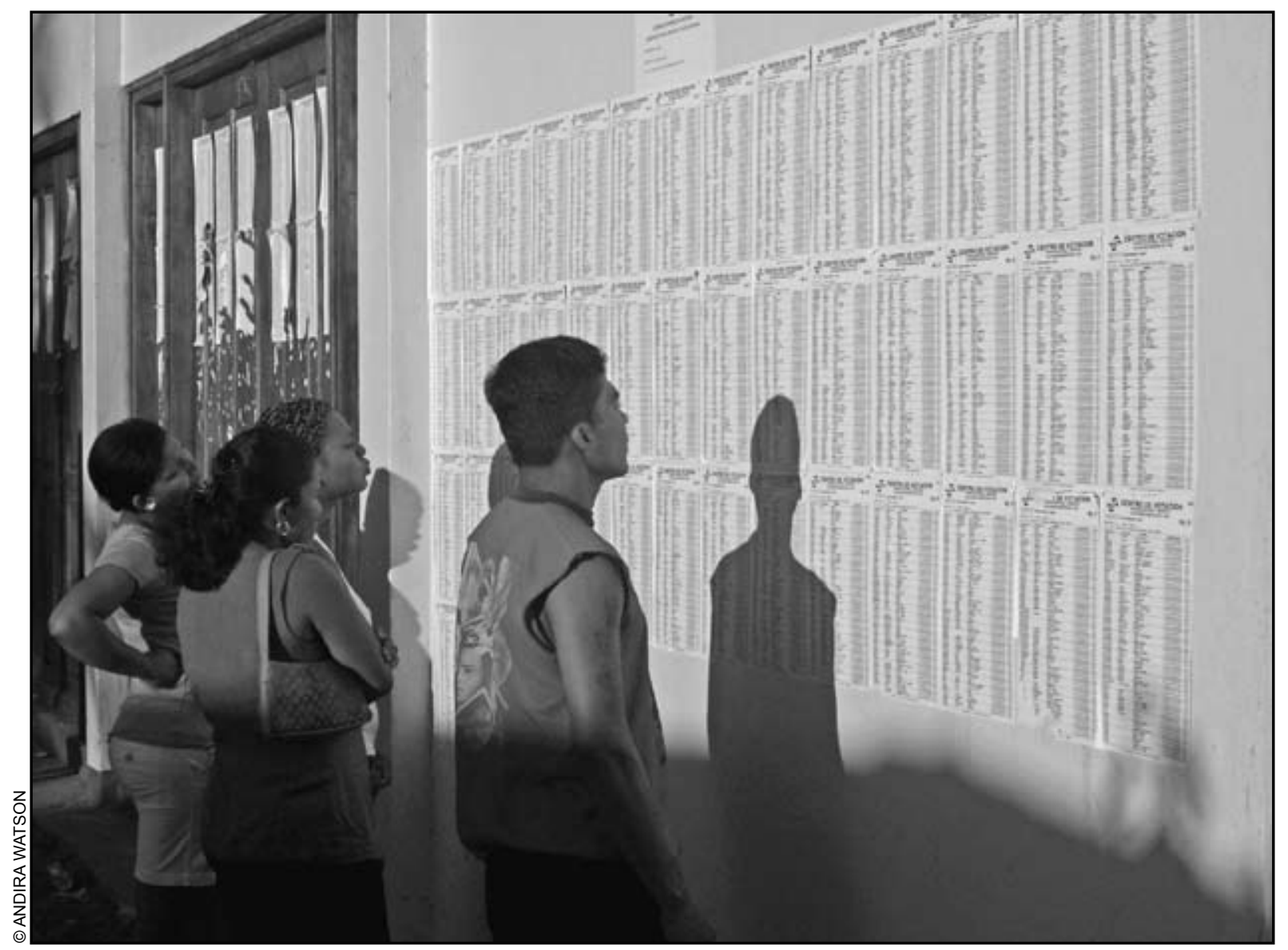

Verificándose en el padrón. Bilwi, enero 2009.

\section{ROSITA: 38 JRV Reportadas}

Ventaja del FSLN. Esta no es una sorpresa electoral ya que era una plaza con relativa hegemonía liberal, la cual el FSLN perdió en 2004 por una diferencia de 250 votos, se esperaba una votación cerrada y al final cambió de manos a favor del FSLN.

Tabla. 5

\begin{tabular}{|l|c|c|}
\hline $\begin{array}{c}\text { Partidos y/o } \\
\text { Alianzas de } \\
\text { Partidos }\end{array}$ & $\begin{array}{c}\text { Votos válidos } \\
\text { por partido }\end{array}$ & $\%$ \\
\hline PLC & 2,481 & $\mathbf{4 1}$ \\
\hline FSLN & 3,486 & $\mathbf{5 7}$ \\
\hline PAMUC & 20 & $\mathbf{0 . 3}$ \\
\hline PRN & 51 & $\mathbf{1}$ \\
\hline YATAMA & 1 & $\mathbf{0 . 0 2}$ \\
\hline ALN & 32 & $\mathbf{1}$ \\
\hline AC & 5 & $\mathbf{0 . 0 9}$ \\
\hline
\end{tabular}

* Elaboración propia de IPADE
BONANZA: 29 JRV Reportadas

Este resultado no es ninguna sorpresa, esta ha sido una plaza fuerte del FSLN, quien aquí va unido con YATAMA y sectores indígenas mayangnas.

Tabla. 6

\begin{tabular}{|l|c|c|}
\hline $\begin{array}{c}\text { Partidos y/o } \\
\text { Alianzas de } \\
\text { Partidos }\end{array}$ & $\begin{array}{c}\text { Votos válidos } \\
\text { por partido }\end{array}$ & $\%$ \\
\hline PLC & 1,420 & $\mathbf{3 0}$ \\
\hline FSLN & 3,310 & $\mathbf{6 9}$ \\
\hline PAMUC & 6 & $\mathbf{0 . 1}$ \\
\hline PRN & 7 & $\mathbf{0 . 1}$ \\
\hline YATAMA & - & $\mathbf{0}$ \\
\hline ALN & 25 & $\mathbf{1}$ \\
\hline AC & $\mathbf{2}$ & $\mathbf{0 . 0 5}$ \\
\hline
\end{tabular}

* Elaboración propia de IPADE 
SIUNA: 87 JRV Reportadas

Hay una ventaja para el PLC. Esta es una de las plazas más fuertes para el Partido Liberal y su resultado expresa el voto tradicional de sus pobladores.

Tabla. 7

\begin{tabular}{|l|c|c|}
\hline $\begin{array}{c}\text { Partidos y/o } \\
\text { Alianzas de } \\
\text { Partidos }\end{array}$ & $\begin{array}{c}\text { Votos válidos } \\
\text { por partido }\end{array}$ & \% \\
\hline PLC & 7,950 & $\mathbf{5 4}$ \\
\hline FSLN & 6,476 & $\mathbf{4 4}$ \\
\hline PAMUC & 29 & $\mathbf{0 . 2}$ \\
\hline PRN & 51 & $\mathbf{0 . 3}$ \\
\hline YATAMA & - & $\mathbf{0}$ \\
\hline ALN & 98 & $\mathbf{1}$ \\
\hline AC & $\mathbf{2 5}$ & $\mathbf{0 . 1}$ \\
\hline
\end{tabular}

* Elaboración propia de IPADE

\section{WASPAN: 29 JRV Reportadas}

Ventaja para el FSLN, en el sector de Llano Norte y área urbana.

Tabla. 8

\begin{tabular}{|l|c|c|}
\hline $\begin{array}{c}\text { Partidos y/o } \\
\text { Alianzas de } \\
\text { Partidos }\end{array}$ & $\begin{array}{c}\text { Votos válidos } \\
\text { por partido }\end{array}$ & $\%$ \\
\hline PLC & 1,639 & 31.4 \\
\hline FSLN & 2,025 & 38.9 \\
\hline PAMUC & 5 & 0.1 \\
\hline PRN & 37 & 0.7 \\
\hline YATAMA & 1,426 & 27.4 \\
\hline ALN & 70 & 1.3 \\
\hline AC & 4 & 0.08 \\
\hline
\end{tabular}

* Elaboración propia de IPADE

Según datos provisionales, dados a conocer por el CSE, al medio dio del 19 de enero; está plaza la gana el FSLN lo cual ha creado inconformidad en partidarios de Yatama quienes han solicitado una revisión de las actas o recuento lo cual fue negado por el CSE a realizarse en el municipio y trasladaron el material electoral a Puerto Cabezas sede del CSE Regional. Lo anterior provocó protestas y disturbios que fueron controlados por las autoridades. Yatama en Waspan así como el candidato del PLC en Puerto Cabezas han señalado el proceso electoral de estos municipios como "sucios" pero no han aportado evidencias de irregularidades. El CSE ha señalado que usen los recursos legales para resolver dicha inconformidad y el CSE Regional esta realizando su propio recuento o revisión, plazo que se vence el 22 de enero para resolver impugnaciones y recursos.

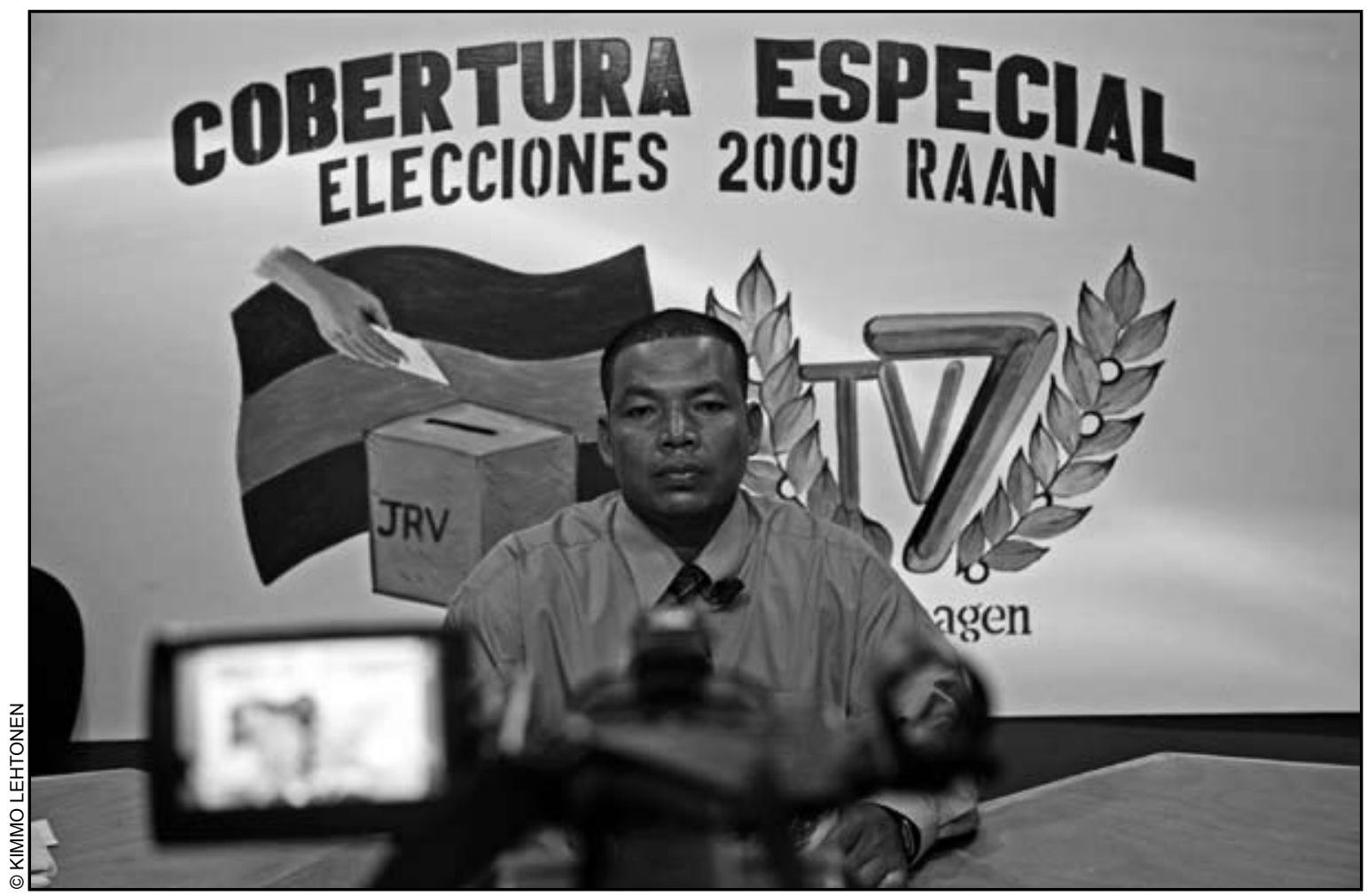

Las elecciones fueron transmitidas en vivo por los dos canales de televisión locales. Bilwi, enero 2009. 


\begin{tabular}{|c|c|c|c|c|c|c|c|c|c|c|c|c|c|c|c|c|c|c|c|}
\hline ১0 & & & & $\stackrel{\rho}{\sigma}$ & దొ & 10 & & \multirow{15}{*}{ 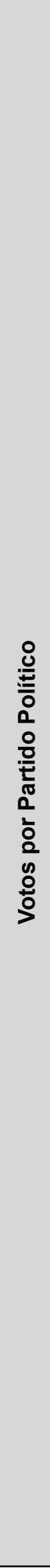 } & $\overline{6}$ & $\hat{\tilde{N}}$ & $\stackrel{N}{O}$ & $\stackrel{\infty}{\sim}$ & & $\begin{array}{l}\stackrel{\infty}{\infty} \\
\infty\end{array}$ & $\underset{\sigma}{N}$ & & & 今े & ○े \\
\hline 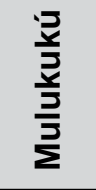 & న్ & 0 & กึ & $r$ & $\begin{array}{l}\mathscr{+} \\
\underset{\infty}{+}\end{array}$ & \&̊ & $\begin{array}{l}\text { N్ } \\
\infty \\
\infty \\
\infty\end{array}$ & & 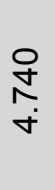 & 유 & $\stackrel{\square}{\square}$ & مొ & & $\begin{array}{l}\text { م) } \\
\text { مִ }\end{array}$ & $\stackrel{\circ}{\square}$ & $\begin{array}{l}\text { \& } \\
+\infty\end{array}$ & $\begin{array}{l}8 \\
\mathbb{0} \\
\stackrel{1}{0} \\
\end{array}$ & $\begin{array}{l}\text { Nิ } \\
\infty \\
\infty \\
\infty\end{array}$ & $\begin{array}{l}\infty \\
\stackrel{0}{0} \\
\infty\end{array}$ \\
\hline ১ீ & คి & 10 & & & คి & 10 & & & 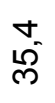 & $\hat{\tilde{C}}$ & $\underset{O}{N}$ & $\tilde{O}$ & & $\begin{array}{l}10 \\
0 \\
0\end{array}$ & $\sigma_{0}^{\circ}$ & & & ๖̊ & ஓ̊ㅇํ \\
\hline $\begin{array}{l}\text { N } \\
\text { N } \\
\text { N } \\
\frac{1}{0} \\
\text { D }\end{array}$ & 우 & $\sim$ & พ & 0 & $\begin{array}{l}\bar{o} \\
\text { ơ }\end{array}$ & $\frac{\omega}{n}$ & $\underset{\sigma}{\stackrel{N}{\sigma}}$ & & 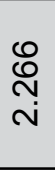 & 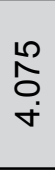 & $\stackrel{m}{\sim}$ & 으 & & $\bar{m}$ & 0 & ơ & $\begin{array}{l}\bar{N} \\
\stackrel{n}{N} \\
\stackrel{n}{\sigma}\end{array}$ & $\underset{0}{\frac{N}{\pi}}$ & 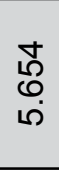 \\
\hline ১0 & & & & $\hat{\sigma}$ & চ & 0 & & & $\stackrel{\sim}{\sim}$ & 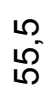 & $\dot{0}_{0}^{\circ}$ & $\stackrel{m}{\sigma}$ & & $\hat{0}$ & $\underset{\sigma}{N}$ & & & Lั & $\mathscr{q}$ \\
\hline $\begin{array}{l}\stackrel{\pi}{\frac{\pi}{D}} \\
0 \\
\mathbb{R}\end{array}$ & ㅇํ & 0 & 윰 & $r$ & $\begin{array}{l}\hat{0} \\
\stackrel{+}{\circ}\end{array}$ & 呫 & $\begin{array}{l}\mathbb{Z} \\
\text { ó } \\
\stackrel{0}{0}\end{array}$ & & 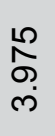 & 岕 & ले & $\stackrel{\stackrel{D}{\sim}}{\sim}$ & & $\widetilde{\mho}$ & $\stackrel{20}{\sim}$ & \begin{tabular}{l}
$\hat{0}$ \\
\multirow{0}{0}{} \\
$\sigma$
\end{tabular} & $\begin{array}{l}\text { ठ } \\
10 \\
\infty \\
\infty\end{array}$ & 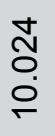 & $\begin{array}{l}\infty \\
\stackrel{\infty}{+} \\
\infty\end{array}$ \\
\hline ১ீ & ه & $N$ & & $\hat{0}$ & ดे & $\nabla$ & & & م & $\frac{0}{\dot{\gamma}}$ & $\stackrel{N}{0}$ & $\begin{array}{r}+ \\
0\end{array}$ & & $\begin{array}{l}0 \\
0\end{array}$ & $\underset{0}{N}$ & & & مْم & $\stackrel{10}{8}$ \\
\hline$\frac{\text { D }}{5}$ & $\underset{\&}{\stackrel{\Xi}{*}}$ & $m$ & $\mathcal{F}$ & $r$ & $\begin{array}{l}\stackrel{1}{\infty} \\
\infty \\
\stackrel{0}{N}\end{array}$ & 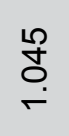 & $\begin{array}{l}\text { ָे } \\
\text { ন্ }\end{array}$ & & $\begin{array}{l}\frac{9}{\pi} \\
\stackrel{m}{\square}\end{array}$ & $\begin{array}{l}\text { O } \\
\text { के }\end{array}$ & $\stackrel{\infty}{+}$ & 음 & & เి & ட் & $\begin{array}{l}\stackrel{n}{\infty} \\
\stackrel{\infty}{N} \\
\end{array}$ & 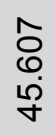 & 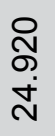 & $\begin{array}{l}\hat{\infty} \\
0 \\
0 \\
0\end{array}$ \\
\hline dீ & $\underset{\infty}{\infty}$ & $\stackrel{\infty}{\leftarrow}$ & & $\stackrel{m}{\forall}$ & ఠै & $\nabla$ & & & $\stackrel{\overbrace{}}{\mathcal{N}}$ & $\stackrel{\infty}{\text { N }}$ & م' & $m^{m}$ & 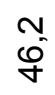 & $\bar{i}$ & $\widetilde{N}$ & & & مص & $\stackrel{\rho}{+}$ \\
\hline 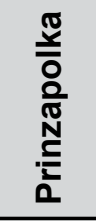 & 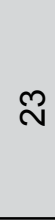 & 10 & $\stackrel{\infty}{N}$ & $r$ & $\begin{array}{l}\text { N్} \\
\text { m} \\
\text { m. }\end{array}$ & $\underset{\sim}{\simeq}$ & ஸे & & ญ઼ & ๙ึ & $\stackrel{\infty}{\leftarrow}$ & 으 & $\begin{array}{l}\text { 웜 } \\
\text { مִ }\end{array}$ & $\bar{\lambda}$ & 0 & लָ & $\underset{⿱ 亠 乂}{+}$ & 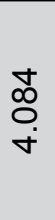 & $\begin{array}{l}\text { ले } \\
\text { m} \\
\text { m. }\end{array}$ \\
\hline ১ீ & & & & $\stackrel{n}{\sim}$ & ⿵̆ & $m$ & & & $\begin{array}{l}m \\
\mathfrak{D}^{0}\end{array}$ & $\begin{array}{l}10 \\
0 \\
0 \\
ల\end{array}$ & 0 & $t_{0}^{\circ}$ & $\begin{array}{l}\text { o } \\
\text { ס }\end{array}$ & $\bar{m}$ & $\underset{\sigma}{N}$ & & & م & $\stackrel{10}{8}$ \\
\hline 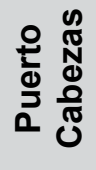 & $\stackrel{\sim}{m}$ & & ָָ & $\sim$ & $\begin{array}{l}m \\
\substack{n\\
} \\
\stackrel{\sim}{N}\end{array}$ & న & $\begin{array}{l}\text { \% } \\
\text { N̦ } \\
\text { N }\end{array}$ & & $\frac{\widetilde{\sigma}}{\oplus}$ & চ & $\bar{m}$ & $\hat{\lambda}$ & 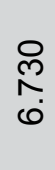 & ర్రి & $\stackrel{\infty}{m}$ & $\begin{array}{l}m \\
\stackrel{\infty}{\infty} \\
\stackrel{N}{N}\end{array}$ & 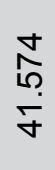 & $\begin{array}{l}\text { Oे } \\
\text { ○ } \\
\text { Nె }\end{array}$ & $\begin{array}{l}\Gamma \\
10 \\
\infty \\
\infty\end{array}$ \\
\hline ১ீ & $\stackrel{10}{\wedge}$ & $\stackrel{\sim}{\sim}$ & & & ద & $N$ & & & $\stackrel{\sim}{\sim}$ & ㅇ & $\underset{0}{N}$ & 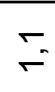 & $\begin{array}{l}0 \\
\text { N }\end{array}$ & $\begin{array}{l}\infty \\
0^{-}\end{array}$ & $\bar{\sigma}$ & & & $\stackrel{10}{+}$ & นొم \\
\hline $\begin{array}{l}\frac{E}{\pi} \\
\frac{0}{0} \\
\frac{0}{0} \\
\frac{\pi}{3}\end{array}$ & ฮ઼ & $\bar{\sim}$ & ఝ్ర & ' & 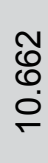 & م & 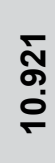 & & $\begin{array}{l}\infty \\
\infty \\
0 \\
\\
\text { N }\end{array}$ & $\begin{array}{l}\infty \\
\stackrel{0}{0} \\
\stackrel{\sigma}{*}\end{array}$ & ㅇ & $\stackrel{\nwarrow}{\sim}$ & $\underset{⿱ 丶}{\stackrel{+}{+}}$ & ○ & $\mp$ & 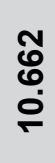 & $\begin{array}{l}\overline{\text { J }} \\
\dot{\sim}\end{array}$ & 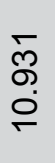 & 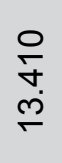 \\
\hline 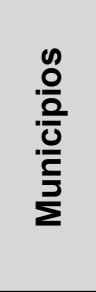 & 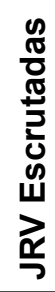 & 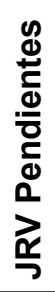 & 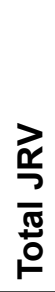 & 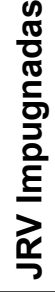 & 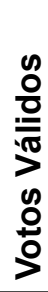 & $\begin{array}{l}\text { on } \\
\frac{0}{3} \\
\Sigma \\
0 \\
0 \\
\stackrel{0}{0}\end{array}$ & 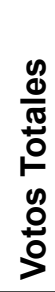 & & U. & $\begin{array}{l}z \\
\text { జ } \\
\end{array}$ & $\frac{u}{2}$ & $\begin{array}{l}z \\
\text { zo } \\
\underline{Q}\end{array}$ & $\frac{\sum_{0}}{\frac{\mathbb{S}}{5}}$ & $\underset{4}{2}$ & U & 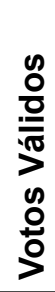 & 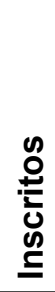 & $\begin{array}{l}\frac{0}{0} \\
\frac{0}{0} \\
\frac{0}{0} \\
\frac{0}{0} \\
\frac{0}{\pi} \\
0\end{array}$ & 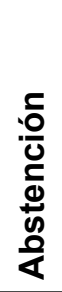 \\
\hline
\end{tabular}




\begin{tabular}{|l|cc|}
\hline \multicolumn{3}{|c|}{ TOTALES } \\
\hline Total Padrón & 167.371 & $100 \%$ \\
\hline Participantes & 88.531 & $53 \%$ \\
\hline Abstención & 78.840 & $47 \%$ \\
\hline
\end{tabular}

\begin{tabular}{|c|c|c|}
\hline \multicolumn{3}{|c|}{$\begin{array}{c}\text { Porcentaje de Juntas } \\
\text { Impugnadas }\end{array}$} \\
\hline Escrutadas & 513 & $100 \%$ \\
\hline Pendientes & 31 & $53 \%$ \\
\hline Impugnadas & 6 & $47 \%$ \\
\hline
\end{tabular}

* Cuadros elaborados por IPADE en base a datos de Informe de CSE del 19 de enero 2009.

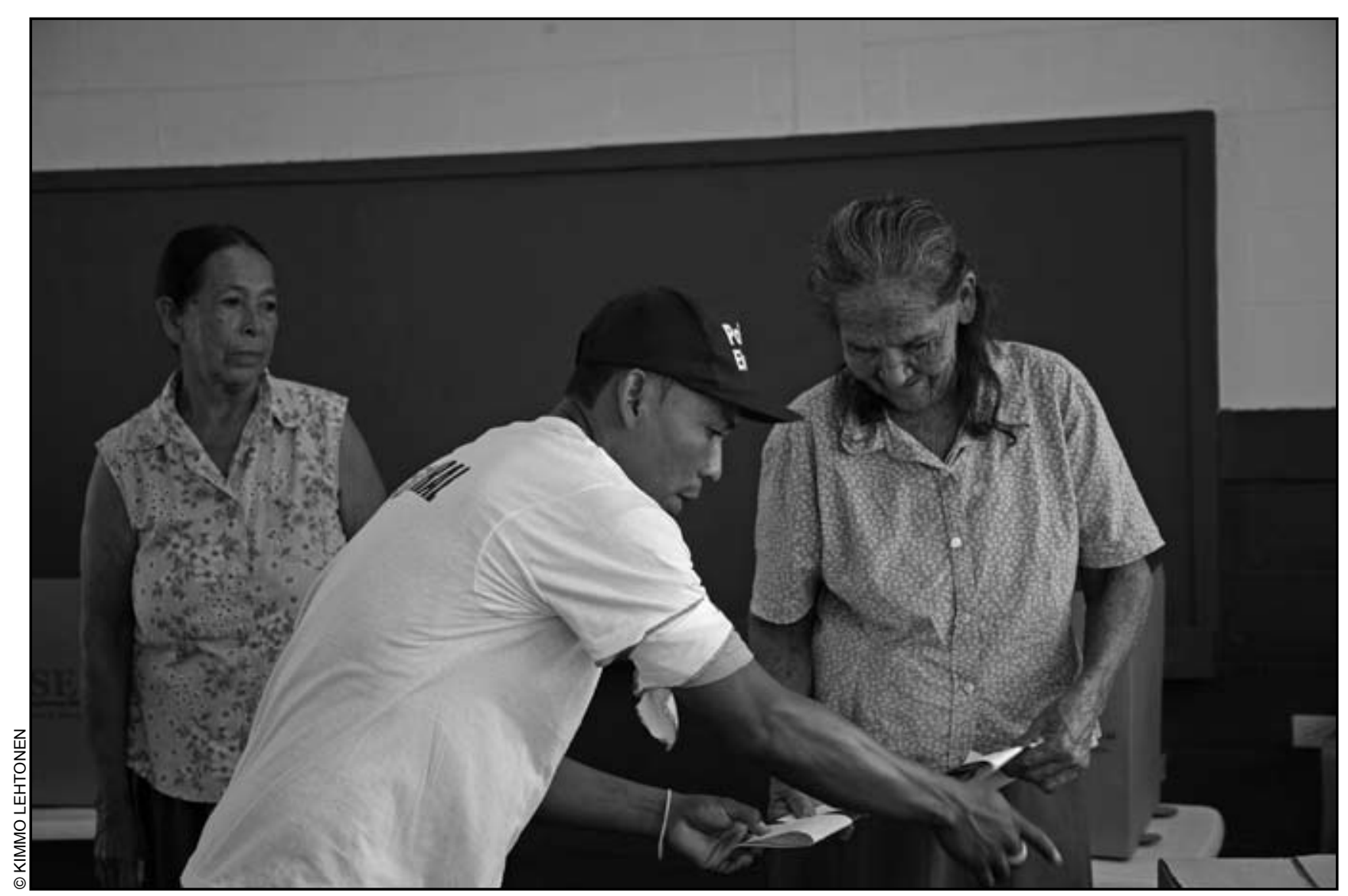

Depositando voto Tuapi, enero 2009.

\section{Conclusiones.}

a) El proceso de votación en estas elecciones municipales de la RAAN se desarrolló sin mayores incidentes y sin la presencia de irregularidades que indiquen a la fecha alteración de la voluntad de los electores. Del cuadro anterior se desprende que el FSLN gana 4 municipios (Waspán, Puerto Cabezas, Bonanza y Rosita), el PLC gana Siuna y Mulukukú y Yatama gana Prinzapolka.

b) La afluencia de ciudadanos a votar, refleja el comportamiento histórico de la región, en cuanto a la participación de 88,531 electores, con un nivel de abstención que oscila entre un 47 a 50 por ciento. c) El comportamiento de los votos nulos se mantiene en los índices normales de $4.15 \%$ variando entre un $2 \%$ al $6 \%$ en diferentes municipios.

c) Se presentaron exclusiones de electores por la no aplicación del artículo 41 de la Ley Electoral, en un pequeño porcentaje de JRV.

d) El Centro Nacional de Cómputo fue cerrado a las 11 de la noche del día 18 , retirando a los fiscales nacionales, lo que provocó inquietud entre los miembros de los partidos políticos. Sin embargo, funcionarios del Centro Nacional de 
Cómputo explicaron que esto había sido un acuerdo previo con todos los Partidos, debido a la afluencia de los datos que entraban de los municipios, pero que se restablecería el día 19 por la mañana.

e) La página Web del CSE no reporta los resultados JRV por JRV, sin embargo, a diferencias de procesos anteriores, por esta vez el CSE cumplió la ley, dando a conocer en conferencias de prensa, el total de votos depositados, votos validos por partido y votos nulos, brindando la información de forma completa, lo cual no ocurrió en el caso del resto de elecciones municipales realizadas el 9 de noviembre del 2008.

f) Los resultados dados a conocer sobre votos nulos, muestran una proporción histórica entre el $2 \%$ y el $5 \%$ en cuanto a los votos anulados en JRV, lo cual establece una diferencia sustantiva con los resultados de las elecciones municipales que se dieron en el Pacífico donde este porcentaje alcanzó incluso el 10\%.

g) IPADE considera que el CSE cumplió con la obligación elemental de garantizar la organización, la logística y las condiciones de transparencia que la Ley Electoral establece.

Al no tener evidencia de que se han producido las irregularidades que caracterizaron las cuestionadas elecciones municipales en un tercio de los municipios del Pacífico (exclusión masiva de fiscales de JRV y CEM, cierre temprano de JRV, impedimento de ejercicio del voto a miles de electores, escrutinio sin presencia de fiscales del segundo partido político, no acreditación de observadores, anulación masiva de votos, impugnaciones generalizadas de JRV, alteración de sumas aritméticas en Centros Electorales Municipales, ausencia de información sobre miles de

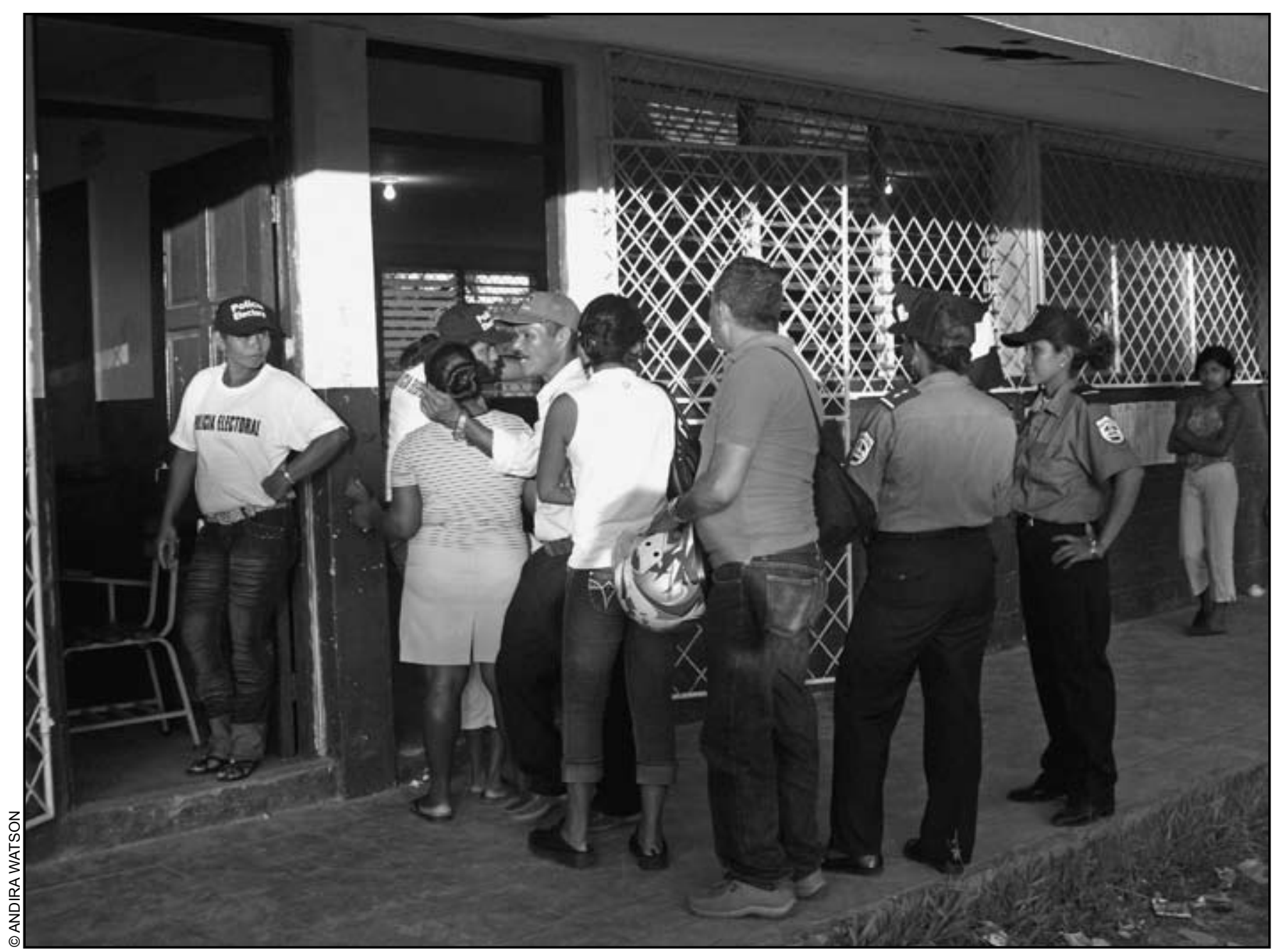

Haciendo fila para votar, Bilwi, enero 2009. 
votos en la capital; etc.), valoramos que las elecciones municipales en los 7 municipios de la RAAN se ajustan en lo general a las garantías y estándares tradicionales de calidad y se han desarrollado con normalidad.

h) Al producirse cuestionamientos sobre los resultados preliminares dados a conocer por el CSE en dos de los municipios (Waspan y Puerto Cabezas), por dos de los partidos participantes, las autoridades electorales están en la obligación de canalizar y dar repuesta en tiempo y forma a los recursos legales interpuestos por Yatama y PLC si así los han hecho, para despejar cualquier duda sobre los resultados y fortalecer la confianza de los electores y actores políticos en el proceso. Los partidos políticos por su parte están obligados a suministrar las evidencias o pruebas que soportan sus reclamos.

i) Asimismo, el CSE debe resolver en tiempo y forma los seis (6) recursos de impugnación existentes y dar a conocer los resultados finales del escrutinio de forma completa.

j) IPADE felicita la labor realizada por el pueblo costeño al acudir a votar de forma cívica y pacífica, a las autoridades de la Policía Nacional y del Ejército al garantizar las condiciones de seguridad, logística y orden público del proceso electoral; a los partidos políticos participantes y a la ciudadanía que integro el personal de JRV y CV garantizando el proceso electoral.

k) Nuestro reconocimiento a la red de voluntarios y voluntarias que pese a nos ser acreditados como observadores, realizaron una labor valiosa para el proceso electoral que posibilitó informar a la ciudadanía sobre el desarrollo y resultados de estas elecciones de la RAAN, recopilando una base de datos paralela al CSE y a los partidos políticos, garantizando la independencia, neutralidad y profesionalismo que caracteriza a la observación electoral de IPADE.

1) Nuestro agradecimiento a los medios de comunicación social que con su despliegue en el territorio y cobertura regional y nacional han contribuido a informar al país de forma objetiva y profesional sobre las incidencias del proceso electoral municipal en la RAAN.

Managua, 21 de enero de 2009.

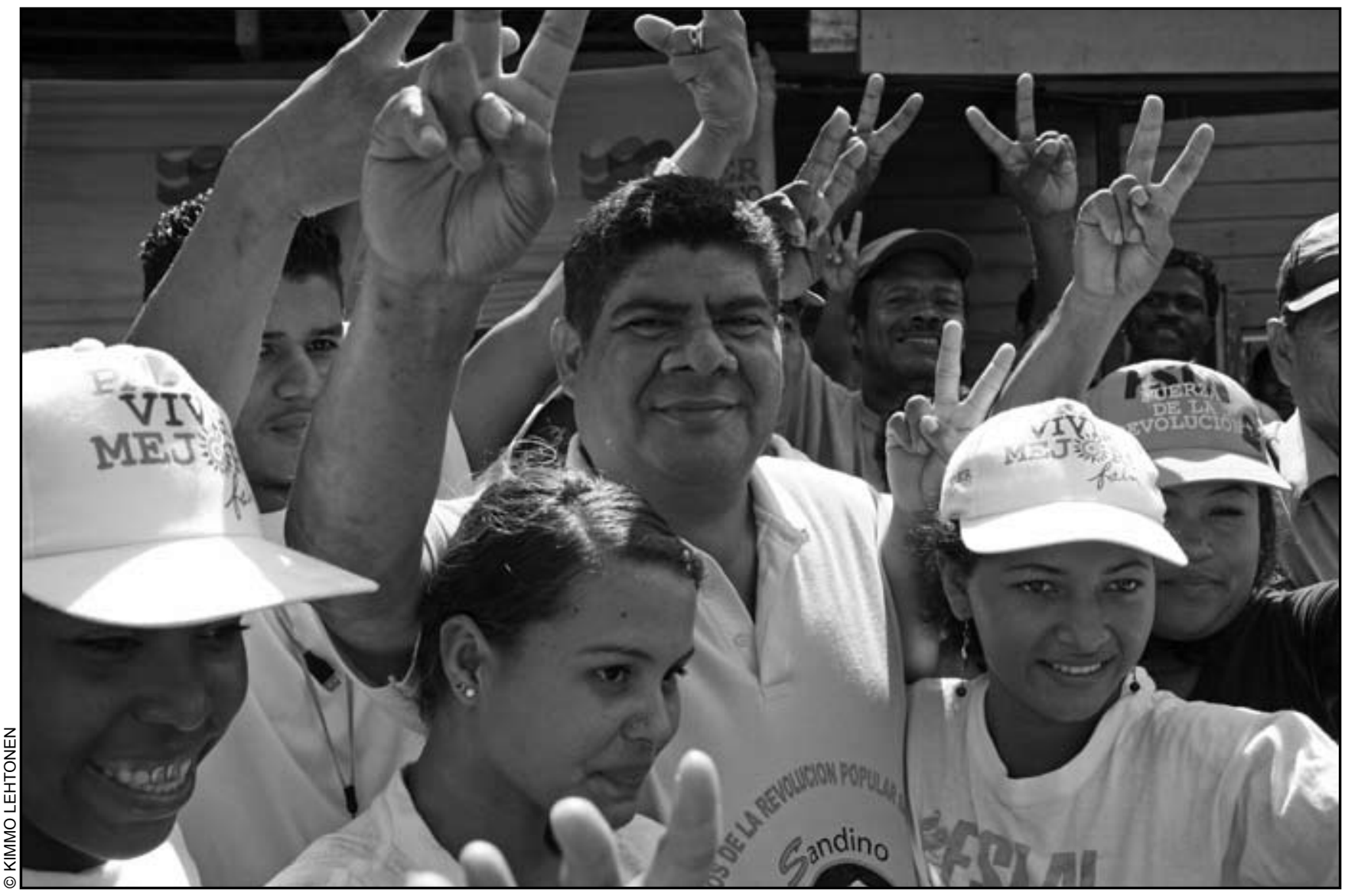

Guillermo Espinoza, nuevo alcalde de Puerto Cabezas. Enero 2009. 